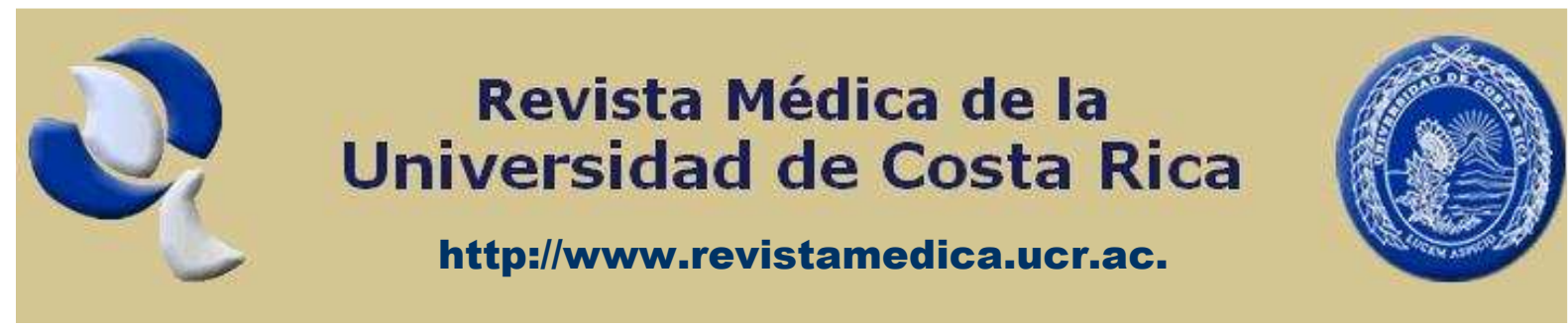

Investigación original

\title{
ESTUDIO DE UTILIZACIÓN DE BENZODIAZEPINAS EN EL ÁREA DE SALUd DE CARTAGO (CCSS), DURANTE EL PERÍODO DE FEBRERO 2007 A ENERO 2008.
}

\author{
Masís Jiménez, María Claret.
}

Servicio de Farmacia. Área de Salud Turrialba-Jiménez. Caja Costarricense de Seguro Social, Turrialba, Cartago, Costa Rica.

\section{Resumen}

El objetivo del presente estudio es describir y analizar el patrón de utilización de benzodiazepinas (Diazepam, Lorazepam y Clonazepam) en el Área de Salud de Cartago (ASC), durante el período de febrero de 2007 a enero de 2008.

Los datos de consumo de benzodiazepinas se obtuvieron directamente de las recetas en las cuales fueron prescritas, por los médicos del Área de Salud de Cartago. Los datos se expresaron en dosis diarias definidas por mil habitantes y día (DHD).

Como parte de los resultados se determinó que una mayoría significativa de prescripciones de benzodiazepinas corresponden a pacientes del sexo femenino. Por otra parte, el grado de utilización de benzodiazepinas fue bastante regular durante el período en estudio, siendo Lorazepam la de mayor demanda. No obstante, en relación con el EBAIS de adscripción de los pacienes, existe gran variabilidad en la utilización de las tres benzodiazepinas analizadas, lo cual se evidencia en los altos consumos en algunos EBAIS o muy bajos en otros.

Tales resultados conllevan a concluir que existe la necesidad de llevar a cabo otros estudios de utilización de benzodiazepinas, que profundicen en el análisis de estos hechos, tanto en el Área de Salud de Cartago como en otras Áreas, de manera que se posibilite la realización de comparaciones.

Palabras clave: Benzodiazepinas, utilización de medicamentos.

Revista electrónica publicada por el Departamento de Farmacología de la Escuela de 
Recibido: Febrero 2009. Aprobado: Marzo 2009. Publicado: Marzo 2009.

\section{Abstract}

The objective of this study is to describe and to analyze the pattern of utilization of benzodiazepines (Diazepam, Lorazepam and Clonazepam) at the Cartago Health Area (ASC), during the period from February, 2007 to January, 2008.

The facts about the consumption of benzodiazepines were directly obtained from the prescriptions which were prescribed for the physicians of the ASC. The information is expressed in defined daily doses per thousand inhabitants and day (DHD).

The results showed up that a significant majority of prescriptions of benzodiazepines belong to patients of the feminine sex. On the other hand, the degree of utilization of benzodiazepines was regular during the analysis period, resulting that Lorazepam was the most demanded. Nevertheless, in relation to the EBAIS of ascription of the patients, great variability exists in the utilization of the three benzodiazepines analyzed, which is demonstrated in the high consumptions of the medicaments in some EBAIS or very low in others.

Such results pointed out that there is a necessity carry out other benzodiazepines utilization studies, and also the analysis of its facts, not only in ASC but in other Healthy Areas, in order to accomplish comparisons.

Keywords: Benzodiazepines, utilization of medicaments.

\section{INTRODUCCIÓN}

Las benzodiazepinas son fármacos psicotrópicos de uso común en el tratamiento a corto plazo de la ansiedad y el insomnio, para tratar las contracturas musculares y la epilepsia, así como también en el manejo de los síntomas de abstinencia etanólica y como premedicación para producir sedación preoperatoria y en procedimientos diagnósticos [1].

Sin embargo, durante su utilización se corren riesgos no sólo debidos a los posibles efectos adversos, sino también a su capacidad de producir tolerancia y dependencia. Esta última es aparente tras 4-6 semanas de tratamiento, y se trata de dependencia tanto física como psicológica. El síndrome de abstinencia ocurre en el $30 \%$ de los pacientes y consiste en ansiedad e insomnio de rebote, temblor y fasciculaciones [2]. Por su potencial adictivo y por sus consecuencias, las benzodiazepinas son consideradas drogas de interés en la problemática del consumo de drogas [3].

En relación con los efectos adversos, las benzodiazepinas de vida media larga, tales como Diazepam, pueden inducir una marcada sedación y falta de coordinación psicomotora, y se han asociado con un incremento en el riesgo de fractura de cadera y de accidentes de tránsito $[4,5]$. Por otra parte, las benzodiazepinas de vida media corta, tales como triazolam y midazolam, aunque se reconocen como más seguras, se asocian con más

Revista electrónica publicada por el Departamento de Farmacología de la Escuela de 
reacciones adversas de tipo psiquiátrico y con fenómenos de rebote. También estas últimas se asocian con un incremento de la proclividad al consumo excesivo y mayor gravedad de la abstinencia después de interrumpir la administración crónica [6].

En general, los usos terapéuticos de una benzodiazepina determinada dependen de su vida media; así, las que resultan útiles como anticonvulsivos tienen una vida media prolongada; es conveniente une vida media breve en el caso de los hipnóticos; en cambio, los agentes que combaten la ansiedad deben tener una vida media prolongada [1].

Por lo anterior, se pretende con este estudio describir el patrón de uso de los fármacos benzodiazepínicos en el Área de Salud de Cartago, con el fin de determinar si existe variabilidad en la prescripción e intuir qué factores podrían trabajarse para minimizar las diferencias.

Los estudios de utilización de medicamentos son "estudios realizados para determinar y explicar el volumen de medicamentos consumidos por una población, sobre la base de una serie de factores de tipo económico-social" [7].

El objeto de estudio de este trabajo es un Área de Salud que corresponde al primer nivel de atención de la Caja Costarricense de Seguro Social; las benzodiazepinas autorizadas para este nivel son Diazepam (tabletas de $5 \mathrm{mg}$, vía oral y ampollas de $10 \mathrm{mg}$, vía intravenosa), Lorazepam (tabletas de $2 \mathrm{mg}$, vía oral) y Clonazepam (tabletas de $2 \mathrm{mg}$ y frasco-gotero 2,5 $\mathrm{mg} / \mathrm{mL}$, vía oral).

\section{MÉTODO Y MATERIALES}

El presente es un estudio descriptivo, longitudinal y retrospectivo. Según la clasificación de los estudios de utilización de medicamentos es un estudio de consumo, los cuales "describen las cantidades y el gasto de los medicamentos dispensados... Son útiles como sistemas de alerta, para detectar desviaciones de consumo, para comparar zonas de salud o médicos, tanto transversal como longitudinalmente, y para aproximar la calidad del medicamento prescrito con datos agregados" [8].

Los datos de consumo de cada especialidad farmacéutica se han obtenido directamente de la revisión de la totalidad de las recetas verdes del Ministerio de Salud, diseñadas para la prescripción de psicotrópicos, alrededor de 5200 recetas emitidas por los veintiún EBAIS del Área de Salud de Cartago, en las cuales se prescribieron benzodiazepinas, las cuales fueron despachadas por la farmacia del Hospital Max Peralta de Cartago durante el período comprendido entre los meses de febrero de 2007 y enero de 2008, inclusive.

Las recetas, al constituir una fuente primaria de información, pueden considerarse como una fuente confiable, puesto que, según las normativas del Ministerio de Salud y de la C.C.S.S, todo fármaco benzodiazepínico de tipo psicotrópico, debe ser prescrito exclusivamente en receta verde; asimismo, estas recetas son custodiadas por los farmacéuticos una vez dispensadas, por lo que para la realización de este trabajo se contó con la totalidad de las mismas, correspondientes al período estudiado.

Revista electrónica publicada por el Departamento de Farmacología de la Escuela de 
Se utiliza la Clasificación ATC (Anatomical Therapeutical Classification) del año 2006, la cual consiste en un sistema de codificación farmacológica estructurado por niveles, y junto con la Dosis Diaria Definida (DDD), conforman el único sistema avalado por la Organización Mundial de la Salud para valorar el uso de fármacos [9].

$\mathrm{Se}$ analizaron los siguientes principios activos, según la clasificación ATC: Lorazepam N05BA06, Diazepam N05BA01 y Clonazepam N03AE01. Los fármacos Lorazepam y Diazepam corresponden al grupo de los psicolépticos-ansiolíticos, mientras que el Clonazepam es clasificado como antiepiléptico, lo cual se relaciona con su indicación principal [10].

Los valores de las DDD propuestos por la OMS son los siguientes: Lorazepam vía oral $=2,5 \mathrm{mg}$; Diazepam vía oral $=10 \mathrm{mg}$, Diazepam vía parenteral $=10 \mathrm{mg}$ y Clonazepam vía oral $=8 \mathrm{mg}[10]$.

a. Tabletas:
Los datos de consumo se han expresado en dosis habitante y día (DHD), con el fin de obtener valores que permitan realizar comparaciones entre diversas poblaciones, según EBAIS del que provienen las recetas, según mes de prescripción de la receta y según sexo de los pacientes; lo cual no podría llevarse a cabo a partir de los No. de DDD, ya que estos no toman en consideración el tamaño de las poblaciones involucradas.

La DHD corresponde a la dosis diaria definida (DDD) por mil habitantes/día. La Dosis Diaria Definida (DDD), es "una unidad técnica internacional de medida de consumo de medicamentos, que corresponde a la dosis diaria media cuando se usa un fármaco en su indicación principal" [11].

Se calculó inicialmente el No. de DDD, mediante las siguientes fórmulas, según la presentación farmacéutica:

No. $\mathrm{DDD}=\underline{\text { No. tabletas prescritas } X \text { No. } \mathrm{mg} \text { por tableta }}$

DDD (mg) X Período analizado (días)

b. Frasco-gotero:

No. $\mathrm{DDD}=$ No. Gotas prescritas X No. mg por gota

DDD en mg $X$ Período analizado (días)

Además, para las formas parenterales se utilizó la siguiente fórmula:

No. $\mathrm{DDD}=\underline{\text { No. } \text { ampollas prescritas } X \text { No. } \mathrm{mg} / \text { ampolla }}$ DDD (mg) X Período analizado (días)

Posteriormente, se sumaron las DDD, en los casos en que exista más de una forma farmacéutica para un mismo principio activo, y se calcularon las dosis diarias definidas por mil habitantes y día (DHD) de la siguiente manera:

Revista electrónica publicada por el Departamento de Farmacología de la Escuela de 
No. $\mathrm{DHD}=\frac{\text { No. }}{\mathrm{DDD}} \times 1000$ Habitantes

No. Habitantes en la población

Para los cálculos se han utilizado las proyecciones y estimaciones de población, obtenidas por Registros Médicos del Área de Salud de Cartago, las cuales pueden considerarse confiables, ya que se toman de los resultados de censos nacionales desarrollados por el Instituto Nacional de Estadística y Censos (INEC).

Esta Área de Salud brinda atención primaria en salud a la totalidad de la población del cantón central de la provincia de Cartago, el cual contaba para el año 2007 con una población de 119651 habitantes [12].

Se excluyen de este análisis las recetas de morbilidad y atención a empleados de esta Área de Salud, debido a que muchos de ellos no viven en el cantón central de Cartago, y por la misma razón tampoco se incluyen las recetas provenientes de la sede del Área de Salud de Cartago, en la cual no existen EBAIS y solamente se realizan labores administrativas, por lo que las mayoría de recetas son prescritas al personal del Área.

\section{RESULTADOS}

Los resultados obtenidos de las prescripciones dirigidas a pacientes adscritos al Área de Salud de Cartago según sexo, muestran una mayoría significativa de prescripciones de benzodiazepinas a pacientes del sexo femenino, con respecto al sexo masculino. Tal como se demuestra en el gráfico 1.
El No. de DHD de Lorazepam prescrito a mujeres es de 3.5 , mientras que el prescrito a hombres es de solo 1.9; por lo tanto, puede interpretarse que casi 4 de cada 1000 mujeres adscritas al Área de Salud de Cartago, consumen en promedio una dosis de 2 mg de Lorazepam al día, mientras que solo 2 de cada 1000 hombres adscritos a esta Área consumen la misma dosis.

Lo mismo sucede con Diazepam y Clonazepam, con la diferencia de que estos tienen DHD menores en comparación con Lorazepam, pero mantienen el mismo patrón de "consumo" por sexo.

Con respecto a la utilización de benzodiazepinas durante el año en estudio, el comportamiento es bastante homogéneo a lo largo del tiempo, como se observa en el gráfico 2 .

El Lorazepam es el que muestra picos de mayor importancia en su utilización, el principal se dio en el mes de octubre con 2,94 DHD, y otros un tanto menores se presentaron en agosto y en abril, ambas con 2,84 DHD. Diazepam presentó un pequeño incremento en la utilización para el mes de agosto con 0,68 DHD, y Clonazepam mantuvo regularidad durante este período, por lo que no se detectan picos de utilización.

Debe destacarse la gran diferencia que existe en la utilización de Lorazepam con respecto a los otros dos fármacos, como se evidencia en el gráfico 2. El No. de DHD de Diazepam se mantiene alrededor de 0,50 y el de Clonazepam entre 0,25 y 0,30 , mientras

Revista electrónica publicada por el Departamento de Farmacología de la Escuela de 


\section{Gráfico 1.}

No. de DHD de benzodiazepinas consumidas durante el período de febrero de $\mathbf{2 0 0 7}$ a enero de $\mathbf{2 0 0 8}$, según sexo

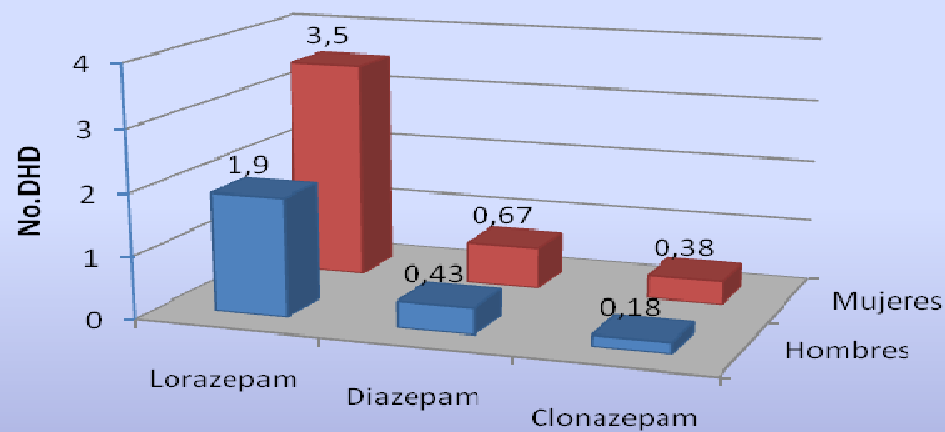

$\square$ Hombres

$\square$ Mujeres

Benzodiazepinas

Fuente: Recetas de psicotrópicos del Área de Salud de Cartago, de febrero 2007 a enero 2008.

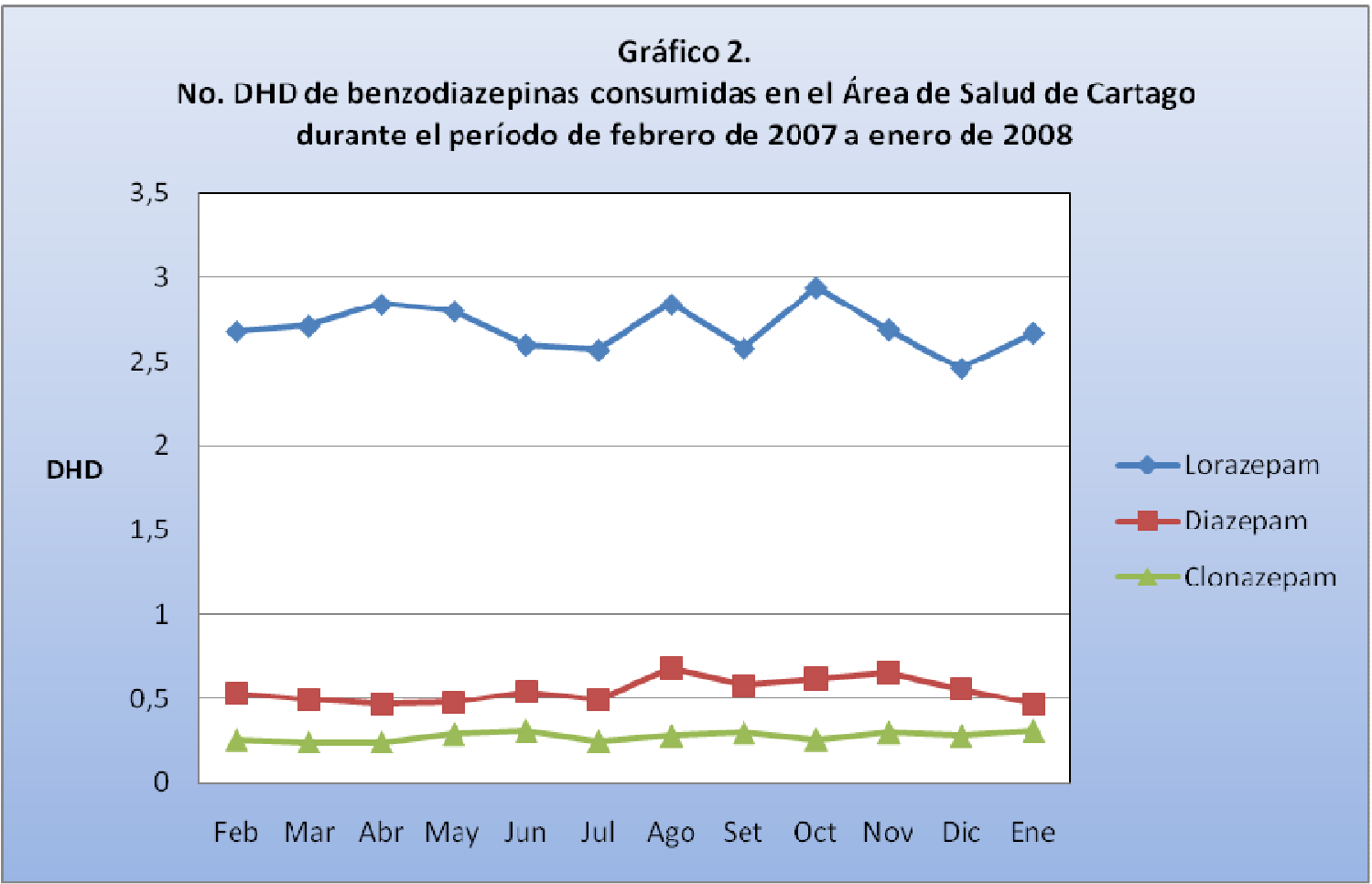

Fuente: Recetas de psicotrópicos del Área de Salud de Cartago, de febrero 2007 a enero 2008.

Revista electrónica publicada por el Departamento de Farmacología de la Escuela de Medicina de la Universidad de Costa Rica, 2060 San José, Costa Rica. (® All rights reserved. 
que a Lorazepam le corresponden de 2,50 a 3,00 DHD durante el período analizado. Esto conlleva a que los picos de utilización sean más detectables para el Lorazepam. Con respecto a la utilización de las benzodiazepinas según EBAIS de adscripción de los pacientes, puede notarse la gran variabilidad que existe entre los diversos EBAIS en relación con las prescripciones. En los siguientes gráficos se observa dicha situación, donde la barra amarilla indica las DHD para el Área de Salud de Cartago, tomando como base la población total.

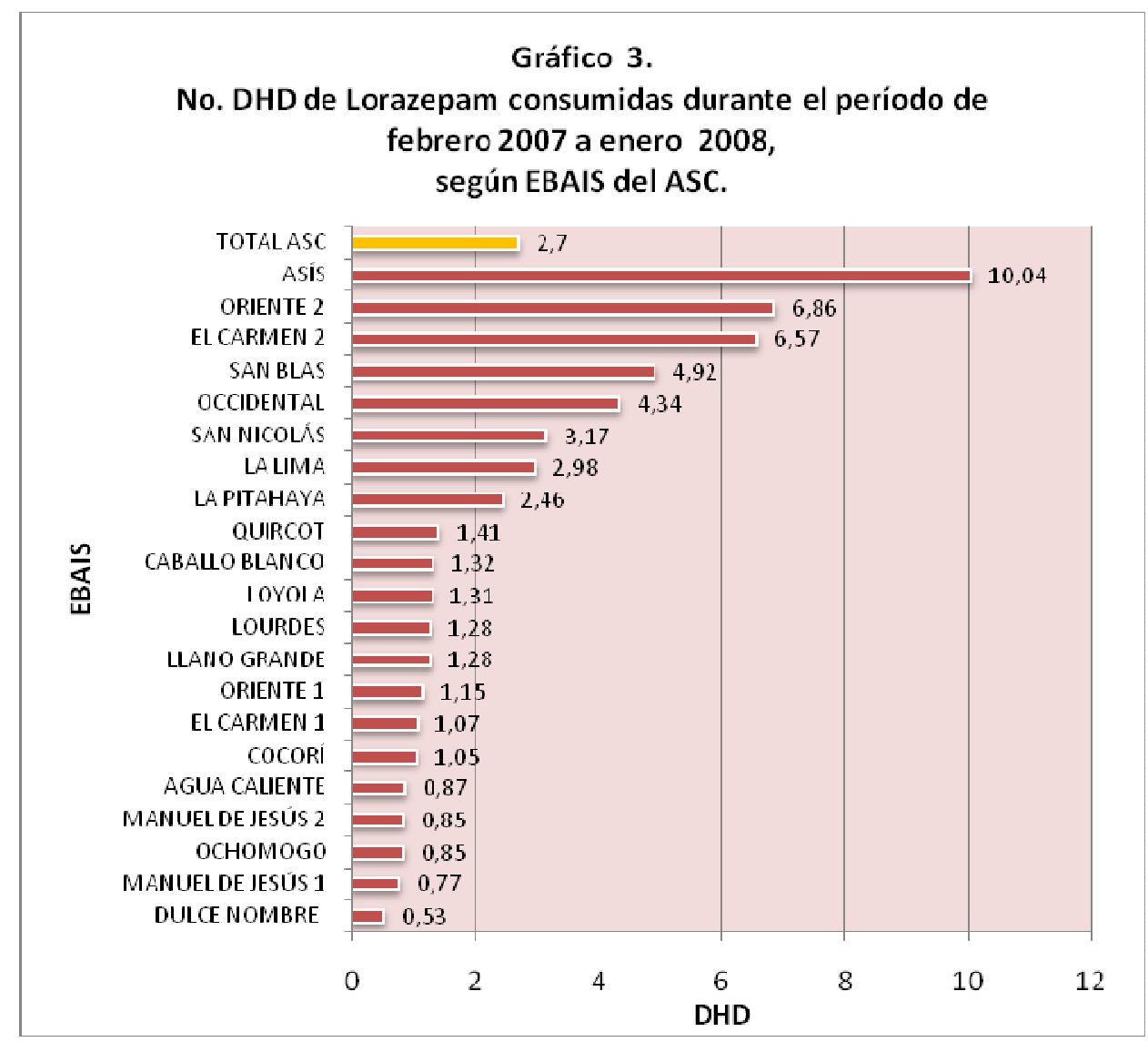

Fuente: Recetas de psicotrópicos del Área de Salud de Cartago (ASC), de febrero 2007 a enero 2008.

En el gráfico 3 se observa que las DHD varían desde 0,53 (EBAIS Dulce Nombre) hasta 10,04 (EBAIS Asís), es decir, en el segundo EBAIS se prescribe Lorazepam 19 veces más que en el primero.
También es importante la diferencia existente entre las prescripciones de EBAIS que atienden a poblaciones muy similares entre sí en cuanto a ubicación geográfica y que incluso atienden en el mismo local, tal es el caso de los EBAIS de El Carmen 1

Revista electrónica publicada por el Departamento de Farmacología de la Escuela de 
y El Carmen 2, con 1,07 y 6,57 DHD respectivamente. Lo mismo ocurre en los EBAIS de Oriente, donde el de Oriente 2 prescribe 6,86 DHD, mientras que el de Oriente 1 solamente 1,15 DHD. En ambos casos el EBAIS 2 prescribió 6 veces más Lorazepam que el EBAIS 1, lo cual demuestra una variabilidad importante en la prescripción de Lorazepam, aún en situaciones muy similares de población. El siguiente gráfico muestra situaciones similares de variabilidad en la prescripción, en este caso de Diazepam.

Las diferencias son importantes de un EBAIS a otro; el EBAIS Occidental es el que más prescribe Diazepam (1,71 DHD), seguido por Oriente 2 (1,62 DHD), Asís (1,41 DHD) y Caballo Blanco (1,11 DHD). Los que menos prescriben este fármaco son: Ochomogo (cero DHD), Manuel de Jesús $2(0,09$ DHD) y La Lima $(0,12$ DHD).

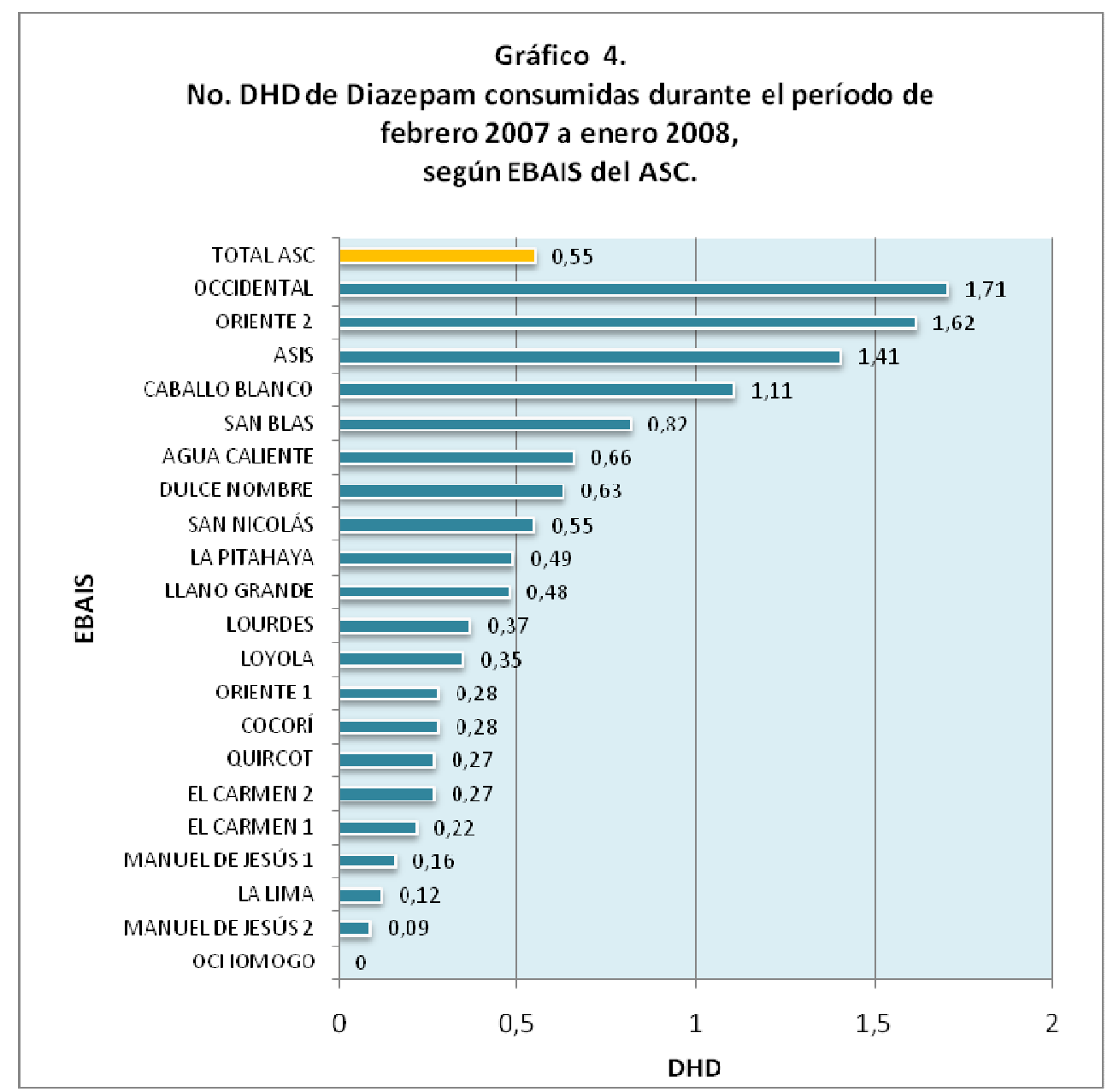

Fuente: Recetas de psicotrópicos del Área de Salud de Cartago (ASC), de febrero 2007 a enero 2008.

Revista electrónica publicada por el Departamento de Farmacología de la Escuela de Medicina de la Universidad de Costa Rica, 2060 San José, Costa Rica. ® All rights reserved. 
Las diferencias son importantes de un EBAIS a otro; el EBAIS Occidental es el que más prescribe Diazepam (1,71 DHD), seguido por Oriente 2 (1,62 DHD), Asís (1,41 DHD) y Caballo Blanco (1,11 DHD). Los que menos prescriben este fármaco son: Ochomogo (cero DHD), Manuel de Jesús 2 (0,09 DHD) y La Lima $(0,12$ DHD).

El gráfico 5 muestra una variabilidad similar a las anteriores en la prescripción de Clonazepam, pero con la particularidad de que un único EBAIS (Oriente 2) prescribió el fármaco en gran proporción al compararlo con el resto de los EBAIS, con 0,96 DHD; el siguiente EBAIS (Asís) presenta una DHD bastante menor de 0,59. El EBAIS que menos prescribe Clonazepam (Manuel de Jesús 2) tiene una DHD de sólo 0,01; el cual es un valor que difiere mucho del resto, es decir, la prescripción del fármaco es mínima.

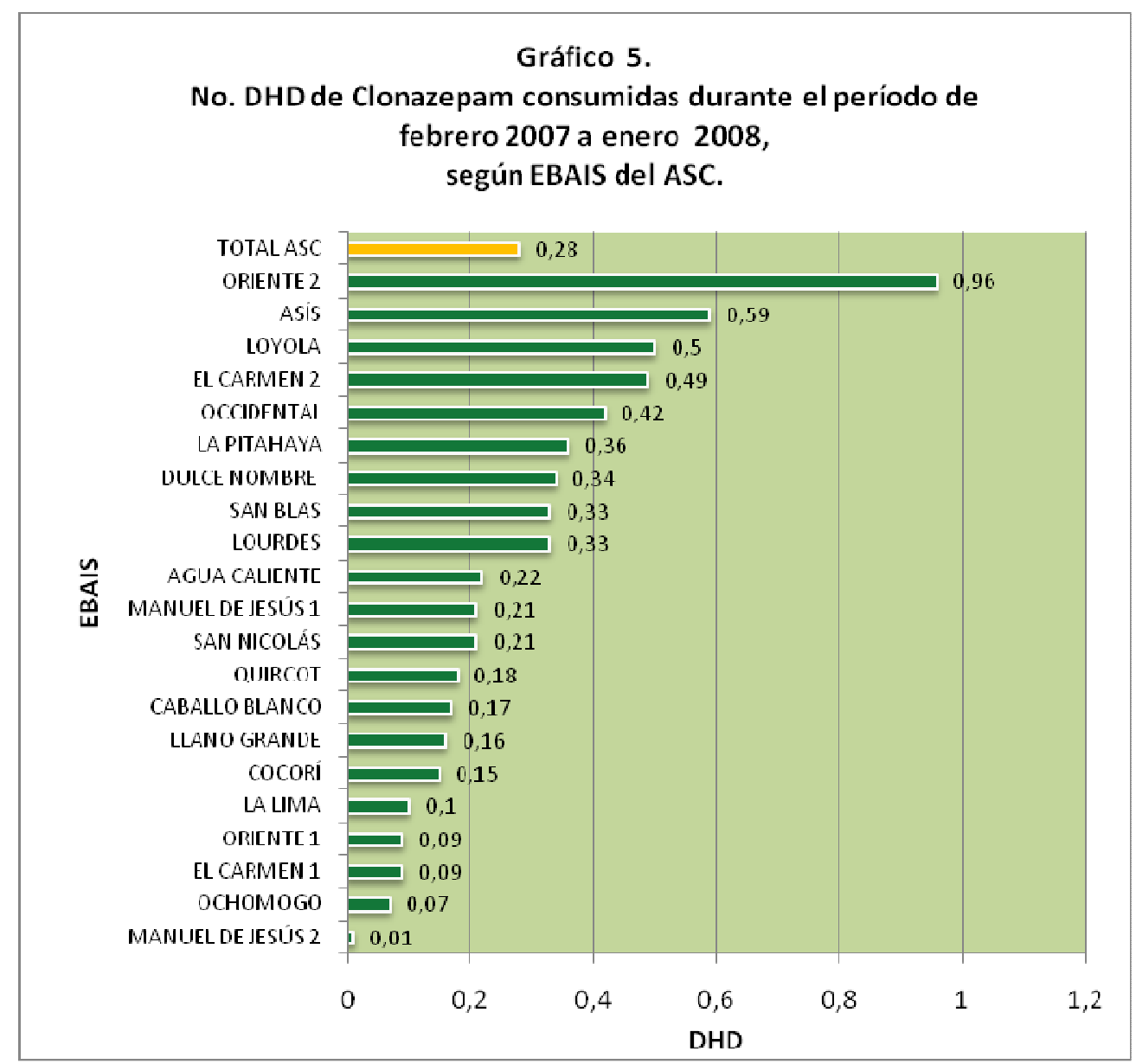

Fuente: Recetas de psicotrópicos del Área de Salud de Cartago (ASC), de febrero 2007 a enero 2008.

Revista electrónica publicada por el Departamento de Farmacología de la Escuela de Medicina de la Universidad de Costa Rica, 2060 San José, Costa Rica. ® All rights reserved. 


\section{DISCUSIÓN}

Antes de analizar los resultados, debe tenerse en cuenta que las cantidades, mal llamadas consumos, en realidad son las cantidades prescritas por los médicos, y no necesariamente las que han sido consumidas por los pacientes, ya que hay que considerar la posible inadherencia a los tratamientos. Asimismo, puede ocurrir una infraestimación de las prescripciones, debido a que las recetas entregadas a los pacientes no siempre llegan a la farmacia para su dispensación, aún menos cuando el paciente es el que resguarda las recetas para presentarlas mensualmente. Por lo tanto, es posible que los resultados anteriormente presentados, en la realidad tengan valores mayores desde el punto de vista de la prescripción.

Por otra parte, puede existir infraestimación de los valores de DHD a nivel poblacional, debido a que se generan prescripciones en el Sistema de Medicina Mixta y en el de Medicina de Empresa, así como de médicos especialistas del Hospital Max Peralta u otros hospitales, los cuales no fueron considerados en esta investigación. Lo anterior, sin embargo, no llega a interferir con los fines de esta investigación, ya que el objetivo primordial del estudio es comparar la prescripción de benzodiazepinas según sexo de los pacientes que son atendidos en EBAIS del Área de Salud de Cartago, según EBAIS de adscripción y según su evolución en el período de estudio.

Es importante destacar que es poco probable que un paciente retire el mismo psicotrópico más de una vez durante determinado mes, debido a que la farmacia cuentan con un sistema computarizado que detecta si ya el paciente ha retirado el medicamento $\mathrm{y}$, de ser así, la receta le es devuelta a la persona. Solamente cabría esta posibilidad si el paciente es atendido en otra Área de Salud o centro de salud y retira el medicamento en otra farmacia, ya que este sistema no se encuentra en red, lo cual no interfiere para los efectos de este trabajo.

Para esta investigación se ha revisado la literatura científica en busca de evidencia acerca de la relación por sexos existente en la incidencia y prevalencia de algunas de las enfermedades comúnmente tratadas con benzodiazepinas, encontrándose que en muchas de estas patologías, esa relación favorece a las mujeres.

Por ejemplo, el trastorno de ansiedad generalizada es ligeramente más frecuente en mujeres que en varones cuando se estudian muestras de centros asistenciales (aproximadamente el $55-60 \%$ de los diagnósticos se efectúan en mujeres); en estudios epidemiológicos de población general la relación de sexos es de dos tercios a favor de las mujeres [13]. El trastorno de angustia sin agarofobia se diagnostica dos veces más en mujeres que en varones, mientras que esta relación es de tres a uno para el trastorno de angustia con agarofobia [13]. Aproximadamente el $75-90 \%$ de los individuos que padecen fobia animal, ambiental y situacional son mujeres [13]. El trastorno obsesivocompulsivo, por su parte, muestra una incidencia similar en varones que en mujeres [3].

Este estudio, en concordancia con la literatura, muestra que el número de DHD de benzodiazepinas prescritas en el Área de Salud de Cartago es mayor en mujeres que en hombres, para los tres fármacos estudiados.

Revista electrónica publicada por el Departamento de Farmacología de la Escuela de 
El comportamiento de las DHD a lo largo del período en estudio, se observa muy regular. Sin embargo, podría indagarse en futuros estudios el pequeño incremento observado en meses como abril, agosto y octubre, para determinar si es esta una tendencia estacionaria y analizar los posibles determinantes de la misma.

El Lorazepam, como era de esperarse, es la benzodiazepina más prescrita en esta Área de Salud, puesto que de las que se disponen en el primer nivel de atención de la C.C.S.S., es la que tiene una vida media más corta en relación con las demás, con una vida media intermedia de $14 \pm 5$ horas, lo que conlleva a menor grado de sedación y déficit psicomotor en los pacientes, que el esperado con Diazepam y aún con Clonazepam [1].

La gran variabilidad observada en el consumo de benzodiazepinas entre los veintiún EBAIS del Área de Salud de Cartago, es lo que más llama la atención, puesto que al tener entre sí una ubicación espacial cercana, sería de esperar que la población tenga ciertas similitudes epidemiológicas y que, por lo tanto, el patrón de prescripción fuera también semejante.

Puede especularse algunas circunstancias por las cuales la prescripción es tan variable entre los diversos EBAIS. Por ejemplo, a pesar de la relativa cercanía entre los EBAIS (pertenecientes todos al cantón central de Cartago), podrían existir algunas diferencias epidemiológicas entre los poblados, en relación con patologías como ansiedad e insomnio; o, bien, diferencias en los factores socioculturales y educativos que influyen en la demanda de medicamentos por parte de la población.
Sin embargo, esta variabilidad también puede estar siendo influida por la formación de grado diferida de los prescriptores, según centro de estudios y época en la que fueron formados como profesionales, o por la existencia o no de educación continua en temas relacionados. Pero también debe tenerse en cuenta la idiosincrasia de los prescriptores al momento de tomar decisiones respecto a la farmacoterapia a instaurar en un paciente dado, donde no sólo influye la formación académica, sino también sus creencias y valores.

Otro factor que puede estar determinando las variaciones en la prescripción de las benzodiazepinas estudiadas, es el predominio de ciertos grupos etáreos en las poblaciones. Por ejemplo, se esperaría que los prescriptores que laboran en poblaciones con mayor cantidad de adultos mayores sean los que más prescriban ansiolíticos e hipnóticos, por la naturaleza del deterioro de los sistemas del organismo que se da en esta etapa, lo que incluye al sistema nervioso.

Como recomendación, los resultados obtenidos a partir de este trabajo cuantitativo, deben invitar a los farmacéuticos a realizar otros estudios que indaguen las causas del comportamiento observado en la prescripción de benzodiazepinas en el Área de Salud de Cartago (estudios cualitativos), otros que realicen intervenciones tendientes a mejorar los hábitos de prescripción (estudios de intervención), a efectuar seguimiento farmacoterapéutico a los pacientes que utilizan benzodiazepinas, y a realizar estudios similares al presente en otras Áreas de Salud, que permitan comparar los resultados y profundizar en su análisis.

Revista electrónica publicada por el Departamento de Farmacología de la Escuela de 
Se espera que lo anterior conlleve a una mejora en la utilización de las benzodiazepinas, al maximizar sus efectos y minimizar los riesgos a los que se expone al paciente con el uso inadecuado de estos fármacos.

\section{CONCLUSIONES}

El hecho que más debe destacarse de los resultados de esta investigación, es la amplia variabilidad que existe entre los diferentes EBAIS en relación con la utilización de las benzodiazepinas, específicamente en las prescripciones, ocurriendo tal variabilidad aún en situaciones poblacionales muy similares.

Por otra parte, durante el período en estudio se determinó que una mayoría significativa de prescripciones de benzodiazepinas fueron dirigidas a pacientes del sexo femenino, lo cual concuerda con la literatura en relación con que las patologías tratadas con benzodiazepinas tienen una mayor prevalencia en las mujeres.

La utilización de benzodiazepinas durante el año en estudio presentó un comportamiento muy regular a lo largo del tiempo, con presentación de pequeños picos de prescripción de Lorazepam en abril, agosto y octubre, y un pequeño pico de prescripción de Diazepam en agosto; la prescripción de Clonazepam fue muy homogénea durante este período.

Se evidenció que Lorazepam es la benzodiazepina de preferencia en las prescripciones de esta Área de Salud, lo cual puede deberse a que es la única de vida media intermedia disponible en este nivel de atención, ya que esta característica hace a este fármaco menos proclive a generar los efectos adversos que se le aducen a las benzodiazepinas de vida media larga como Diazepam y Clonazepam.

\section{AGRADECIMIENTOS}

A la Dra. Mónica Ausejo Segura, Consejería Sanidad, Comunidad de Madrid, España; y a la Dra. Maureen Moulton Allen, Directora de Farmacia del Hospital Max Peralta de Cartago, a ambas farmacéuticas por su valiosa colaboración en la realización de este trabajo.

\section{References}

1. Charney DS, Mihic SJ, Harris RA. Hipnóticos y sedantes. En:. Las bases farmacológicas de la terapéutica (Goodman \& Gilman). Hardman JG, Limbird LE, Gilman AG. (eds.) McGrawHill Interamericana, México, D.F. Décima edición 2003, pp 416-420.
2. Dawson, James; Taylor, Magali; Reide, Peter. Lo esencial en Farmacología. Editorial Elsevier: Madrid, España. Segunda edición, 2003.

3. Sandí L, Díaz A. Consumo de Benzodiacepinas en adolescentes costarricenses. Fármacos. 1996;

Revista electrónica publicada por el Departamento de Farmacología de la Escuela de 
9(1):31-7.

http://www.binasss.sa.cr/adolescencia/B enzodiacepinas.htm. Accesada el $1^{\circ}$ de marzo de 2009.

4. Hemmelgarn B, et al. Benzodiazepine use and the risk of motor vehicle crash in the elderly. JAMA. 1997; 278: 27-31.

5. Ray WA, Griffin MR, Downey W. Benzodiazepines of long and short elimination half-life and the risk of hip fracture. JAMA. 1989; 262: 3303-07.

6. Ashton $\mathrm{H}$. Guidelines for the rational use of benzodiazepines. When and what to use. Drugs. 1994, 48: 25-40.

7. Iñesta García, A. Estudios de utilización de medicamentos, revisión. Madrid: Escuela Nacional de Sanidad; mayo 2007. Documento docente.

8. González, Beatriz et al. Evolución de los estudios de utilización de medicamentos: del consumo a la calidad de la prescripción. Cuadernos Económicos del ICE. 2005; (67):3-11.

9. Saladrigas, María Verónica. El sistema de clasificación ATC de sustancias farmacéuticas para uso humano (The Anatomical, Therapeutic, Chemical Classification System). Panacea: boletín de medicina y traducción. Marzo 2004; 5(15): 58-60.

10. ATC Index with DDDs. WHO Collaboration Centre for Drug Statistics Methodology, 2006.

11. Arnau JM, Vallano A. Estudios de utilización de medicamentos. Medicamentos y salud. 2000, 2:78-82.

12. Caja Costarricense de Seguro Social, Área de Salud de Cartago. Proyecciones de población, año 2007.

13. Ballenger, James; et al. Trastornos de ansiedad. En: Manual diagnóstico y estadístico de los trastornos mentales (DSM-IV). Pichot, P., López, J.J., Valdés, M. (eds.) Masson, Barcelona, España. Cuarta edición 1995, pp 445446.

\section{Correspondencia:}

Ma. Claret Masís Jiménez, Servicio de Farmacia, Área de Salud Turrialba-Jiménez, C.C.S.S.

Correo electrónico: mclareth@yahoo.es Teléfonos: 2532-2283/2531-2885/ 2531-1019.

Revista electrónica publicada por el Departamento de Farmacología de la Escuela de 